\title{
LA IDENTIDAD PERSONAL Y RELIGIOSA EN EL ESPACIO PÚBLICO. UN COMENTARIO DESDE LA OBRA DE CHARLES TAYLOR \\ Diego Rosales Meana*
}

\begin{abstract}
RESUMEN: El objetivo de este trabajo es mostrar cómo se articulan las identidades religiosas en el espacio público según el pensamiento de Charles Taylor. Podría parecer que las expresiones religiosas deben quedar relegadas al ámbito privado. Sin embargo, el multiculturalismo de Taylor permite comprender el espacio público de manera incluyente. En primer lugar, hablaré de las diferentes nociones de "identidad". En segundo, de la identidad religiosa y de su racionalidad. Por último, de la necesidad reconocimiento y de cómo la afirmación de la identidad religiosa debe ser algo más que permitir el culto de los creyentes.
\end{abstract}

PALABRAS ClAVE: Charles Taylor, identidad, reconocimiento, religión, multiculturalismo.
ABSTRACT: The objective of this work is to show Charles Taylor's theory of how religious identities are coordinated in the public scene. It would seem that religious expression should remain relegated to a private environment. However, Taylor's multiculturalism allows an inclusive view of the public space. Firstly, we will address the different notions of identity and then of religious identity and its rationality. Finally, we will approach the need for recognition and how this realization of religious identity should go beyond the tolerance of religious practice.

KEYwORDS: Charles Taylor, identity, recognition, religion, multiculturalism.

* Centro de Investigación Social Avanzada. 


\author{
LA IDENTIDAD PERSONAL Y \\ RELIGIOSA EN EL ESPACIO PÚBLICO. \\ UN COMENTARIO DESDE LA OBRA \\ DE CHARLES TAYLOR
}

\title{
La identidad personal
}

$E_{\text {s normal escuchar hablar de la }}$ identidad en nuestros días. Pero hablar de la identidad e intentar comprender el significado de esta idea es bastante complejo. Desde la filosofía antigua, la pregunta por la identidad ha sido planteada desde la ontología. La pregunta por la identidad del ser ha constituido un problema filosófico. ¿A es igual a "A"? ¿Cómo explico, entonces, el cambio? Ya desde Parménides, Platón y Aristóteles, pasando por Tomás de Aquino, Leibniz, Hegel, Frege, Husserl y hasta Quine, Searle, Parfit o Merleau-Ponty el problema de la identidad personal ha constituido una pregunta importante. Por otra parte, también la psicología se ha preguntado por la identidad del yo y el modo de su génesis y constitución. Freud, Lacan, Erikson y Rogers, por mencionar solamente algunos, han afrontado esta cuestión. Sin embargo, hay un tercer punto de vista que resulta ser una suerte de combinación de ambos planteamientos: la identidad en el sentido antropológico. Aquí ya no se pregunta si un ente es idéntico a otro ente, o cuál es la génesis de la personalidad, sino que se trata de responder a la pregunta "¿quién soy?"

Desde ahí quiero abordar el problema de la identidad, desde donde el mismo Taylor lo hace. En este sentido, la identidad es la definición que un agente humano hace de sí mismo, 
aquella idea que tenemos de nosotros mismos y que vamos configurando a lo largo de la vida. La identidad nos da un marco de referencia, estabilidad y seguridad, pues nos sitúa en el mundo. ¿Qué es lo que quiero decir con esto? "No resulta fácil de definir. Pero se podría decir que mi identidad me define de alguna manera el horizonte de mi mundo moral. A partir de mi identidad sé lo que resulta verdaderamente importante para mí y lo que resulta menos importante, sé lo que me atañe profundamente y lo que tiene una significación menor". ${ }^{1}$ Mi identidad me sitúa en mi mundo moral, me orienta a actuar y a situarme en el ámbito social. Ahora bien, hay que evitar en este caso entender 'mundo moral' como aquello que es bueno y que es malo, como aquellas normas que me dicen qué está bien hacer y qué mal. El concepto de "lo moral" es entendido por Taylor en un sentido mucho más amplio y comprensivo: "Quiero también centrarme en lo que subyace a nuestra dignidad o en cuestiones que hacen de nuestra vida algo significativo. Esto podría ser considerado como cuestiones morales en un sentido, pero muchas están demasiado referidas a la autocomprensión o a lo que tiene que ver con nuestros ideales como para ser consi-

${ }^{1}$ Charles Taylor, "Identidad y reconocimiento", en Revista Internacional de Filosofía Politica, 1997, v.7, pp. 10-9. deradas como asuntos morales en el léxico de muchas personas. Se refieren, más bien, a lo que hace que una vida valga la pena". ${ }^{2}$ La identidad es, en este sentido, el horizonte moral que define a mi vida como algo valioso, y que constituye los criterios para dirigir mis acciones y mis deseos. Un "horizonte moral", entonces, podría ser definido como la serie de nociones, conceptos e ideas sobre el mundo y sobre la vida que conforman los criterios bajo los cuales oriento mi vida.

La identidad, considerada de esta forma, se genera a partir de dos factores: lo social, que es extrínseco al yo, y lo personal, que es intrínseco. Lo social está configurado por los factores que el yo no decide acerca de su persona: color de piel, sexo, lengua, país de nacimiento o la educación que ha recibido, por poner algunos ejemplos. Lo personal, o intrínseco al yo, se refiere a todos los factores internos que diferencian al yo de los demás y que lo hacen ser único e irrepetible. "Esto nos coloca en un segundo contexto del discurso de la identidad, que la entiende como algo personal, potencialmente original e inédita y, por consiguiente, inventada o asumida en cierta medida". ${ }^{3}$

${ }^{2}$ Charles Taylor, Sources of the Self. The Making of the Modern Identity, 1989, Cambridge Mass., Harvard University Press, p. 4.

${ }^{3}$ Charles Taylor, "Identidad y reconocimiento", op. cit., p. 12. 
NOTAS

El primer sentido de identidad, surge históricamente, según Taylor, desde la antigüedad. Esta identidad está conformada por horizontes referenciales extrínsecos al yo y lo definen de manera más o menos universal, como perteneciente a determinado grupo, comunidad o insertándolo dentro de un tipo más o menos general. El segundo sentido es, en cambio, más propiamente moderno. En él se abre el ideal de autenticidad, de originalidad y de completa unicidad. "Hay cierto modo de ser humano que es mi modo. He sido llamado a vivir mi vida de esta manera, y no para imitar la vida de otra persona. Pero esta idea atribuye una importancia nueva a la fidelidad que me debo a mí mismo. Si no me soy fiel, estoy desviándome de mi vida, estoy perdiendo de vista lo que es para mí ser humano". ${ }^{4}$ Esta vuelta de tuerca que va de lo externo a lo interno, se debe según Taylor a una concepción expresivista del ser humano, motivada por el giro moderno hacia la subjetividad, una de cuyas expresiones más importantes es el romanticismo alemán, entre otros factores sociales y culturales. ${ }^{5}$

${ }^{4}$ Charles Taylor, "La política del reconocimiento" en Gutman, Amy (ed.), El multiculturalismo y "la política del reconocimiento", México, FCE, 2009, trad. Mónica Utrilla de Neira, p. 60.

${ }^{5}$ Para una mayor explicación del giro subjetivista y el centramiento en la interioridad del yo en la construcción de la identidad moderna, véase Charles Taylor, Sources of the Self... op. cit., específicamente la segunda parte: "Inwardness", pp.109-207.
La identidad es, en este sentido, objeto de investigación por parte del yo. El yo mismo debe desarrollarla y desenvolverla a lo largo de su vida. Cada quien debe "asumir" su identidad y participar, aunque no de manera completa, en la formación de la idea que tiene de sí mismo en diálogo con los factores externos que le otorgan un horizonte moral. Hay un cruce entre lo dado por el exterior, lo elaborado por el yo, y lo descubierto en sí mismo acerca de sí. La relación entre todo esto va constituyendo el horizonte moral que configura su identidad y que, a su vez, le orienta en aquello que es importante y que hace que su vida sea más valiosa y que valga la pena ser vivida.

En sentido moderno y desde un punto de vista ilustrado, este horizonte moral, esta idea que tiene el yo de sí mismo, debe ser de alguna manera razonable o cumplir con las características de lo que suele denominarse "racionalidad". Las sociedades modernas piden que aquello que los define como tales no se contradiga y que pueda ser razonablemente justificado. Solamente así puede erigirse un verdadero sujeto moderno: en la medida en que su identidad, es decir, su horizonte moral, sea razonable, comprensible y compatible con los valores del ciudadano mayor de edad. El agente moderno se va constituyendo, en primer lugar, de acuerdo 
con lo que piensa que es bueno o valioso; en segundo lugar, justificando esa creencia (dando razones en su favor); y, en tercer lugar, actuando en consecuencia. "La acción no sólo nos permite ver el deseo; es el deseo encarnado en el espacio público". ${ }^{6} \mathrm{El}$ deseo está determinado por el horizonte moral, pues el agente desea aquello que concibe como bueno $\mathrm{y}$, en esa medida, debe traducirse en su desenvolverse y su actuar en el mundo. De esta manera, en términos de Taylor, hay una conexión entre identidad (horizonte moral) y acción en el espacio público. Qué idea tenga yo de mí mismo determinará mi acción y mi actuar en el mundo. Ahora bien, hay que preguntarnos, ¿qué es una identidad religiosa?

\section{La identidad religiosa y la epistemología}

La identidad religiosa, como cualquier otra identidad, es aquella que define nuestros horizontes morales. Incluso, puede decirse que lo religioso no es necesariamente exclusivo de una identidad y que, generalmente, la religión ocupará solamente un lugar más o menos preponderante, según sea el caso, en la identidad de cada per-

${ }^{6}$ Charles Taylor, "La acción como expresión", en La libertad de los modernos, 2005, Buenos Aires, Amorrortu Editores, trad. Horacio Pons, p. 92. sona. Lo específicamente religioso, si bien es extremadamente complejo de definir y delimitar, en términos generales, es un fenómeno según el cual ciertos individuos o comunidades de personas creen en la existencia de un ser superior o en algún modo de trascendencia. La religión es la relación con lo divino, y lo divino significa una realidad trascendente a este mundo. Por supuesto, habría que hacer aquí muchos matices y aclaraciones para que ningún creyente o sector religioso sintiera que esta somera definición es injusta. Por lo pronto, quiero dejarla hasta este punto como hipótesis de trabajo, consciente de que es necesario emprender toda una filosofía y una fenomenología de la religión que dé cabal cuenta del hecho religioso. En cualquier caso, "la religión -dice López Farjeat- constituye un conjunto de creencias y motivaciones, marcos de referencia, normas morales y criterios de acción, que un sujeto asume con la finalidad de dar respuesta a un conjunto de misterios e inquietudes que le han marcado existencialmente". ${ }^{7}$ En esta medida, la religión también ofrece al individuo que se asume religioso una serie de creencias que le dotan de un horizonte moral determinado, es decir, le dota de una identidad.

${ }^{7}$ Luis Xavier López Farjeat, “Arrogancias dispares”, en Conspiratio 2010, núm. 8, año II, p. 54. 
NOTAS

La religión es, la mayoría de las veces, tanto un factor de identidad extrínseco como intrínseco. Es extrínseco por muchas razones: en muchos casos, es transmitida por lo padres; genera un lenguaje y desarrolla un vocabulario propio; incluye costumbres y tradiciones, modos de vestir y de construir, entre muchos otros. Es intrínseco, también, porque toda esta serie de factores generan en el individuo una concepción del mundo y del bien, un criterio de lo que es importante y de lo que vale la pena ser vivido. Por otra parte, la identidad religiosa es un factor intrínseco en la medida en que está en manos del sujeto denegar eso que le ha sido entregado por la tradición, la historia o la familia y, en caso de aceptarlo, lo hará según su propio estilo y su propia personalidad.

En muchos casos, y el mismo Taylor lo sostiene en su comentario a Las variedades de la experiencia religiosa de William James, la religión ofrece al individuo una serie de criterios, conceptos y juicios que no pensaría jamás un sujeto por sí mismo, pues la apertura a la trascendencia le permite pensar en categorías más grandes que las que el propio yo puede producir: "en ciertos dominios, el amor y la apertura nos permiten entender lo que nunca podríamos captar de otro modo, y que no se deduce como la consecuencia normal del pensa- miento". ${ }^{8}$ Sin embargo, a pesar de este tipo de afirmaciones, muchas corrientes del pensamiento ilustrado-liberal denotan las posturas y las creencias religiosas como irracionales o míticas, y no las consideran como una opción justificada para que los individuos formen su identidad.

¿Es razonable que una religión conforme mi identidad? Sin entrar en la discusión, que llevaría bastante más tiempo y espacio del que dispongo aquí, acerca de la racionalidad o razonabilidad de la religión -que, además, sin duda, es discusión de otra área de la filosofía-, lo único que dejaré en claro es que una postura verdaderamente liberal e ilustrada debe pensar que una creencia religiosa podría llegar a ser razonable. En "The Diversity of Goods"("La diversidad de los bienes"), Taylor hace una crítica a las filosofías políticas, sobre todo de corte liberal o utilitarista, porque hacen residir sus evaluaciones morales, en última instancia, en epistemologías empiristas y utilitaristas, con lo que terminan por estrechar los límites de lo que se puede considerar o no moral y racional.

Con la intención de evadir una serie de discusiones metafísicas, supuestamente indecidibles, ciertas corrientes del liberalismo moderno han dejado fuera de la discusión todo

${ }^{8}$ Charles Taylor, Las variedades de la religión hoy, 2003, Barcelona, Paidós, trad. Ramón Vilà Vernis, p. 57. 
sentido de lo moral que no sea cuantificable o científicamente constatable. "En la perspectiva utilitarista, uno validaba una postura ética con evidencia dura. Se contaban las consecuencias de felicidad de una posibilidad, y se decidía por aquella que tenga el total más favorable. Lo que contaba como felicidad humana era considerado como algo conceptualmente no problemático, un dominio de hechos científicamente decidibles". ${ }^{9}$ En este sentido, los formalismos y utilitarismos ofrecen la ventaja de evaluar una posición moral con evidencia dura, sin entrar en discusiones teológicas inverificables, sin discutir intenciones, motivaciones, la cualidad de las acciones o los sentimientos. El utilitarismo y el liberalismo político han tomado estos principio epistemológicos y los han aplicado a la esfera política.

Esto ha permitido, en buena medida, que valores como la autonomía del individuo funcionen como el último criterio de decisión para hacer válida o no una acción: "no importa qué decidas, lo que importa es que lo decidas tú y que no hagas daños a terceros". "Detrás de estas formulaciones derivadas de Kant, descansa una de las más importantes intuiciones de la civilización moderna

${ }^{9}$ Charles Taylor, "The Diversity of Goods", en Philosophical Papers 2. Philosophy and the Human Sciences, 1985, Cambridge, Cambridge University Press, p. 230. de Occidente, la atribución de la personalidad moral: en los asuntos éticos fundamentales, todos los individuos deben contar y deben de hacerlo en el mismo modo. Dentro de esta perspectiva, es un requisito absoluto del pensamiento ético que respetemos a los otros agentes humanos como sujetos de razonamiento práctico en el mismo sentido que nosotros" ${ }^{10}$ Todo agente es considerado igualmente digno y valioso.

Esto trae como ventaja para el razonamiento político la posibilidad de evaluar las posiciones morales desde una perspectiva universal, sin entrar en discusiones cualitativas sobre la identidad. Sin embargo, y cito a Taylor in extenso:

El precio de este formalismo, $\mathrm{y}$ también de la reducción utilitarista, ha sido una severa distorsión de nuestra comprensión del pensamiento moral. Una de las grandes ilusiones que surge de cualquiera de estas reducciones es la creencia de que hay un único y consistente dominio de "lo moral", que hay toda una serie de consideraciones, o un modo de calcular, que determina lo que deberíamos "moralmente" hacer. La unidad de lo moral es una cuestión conceptualmente decidida, en principio, sobre el fundamento de que el razonamiento moral es equivalente al cálculo de consecuencias

${ }^{10}$ Ibid., p. 231-2. 
NOTAS

para la felicidad humana, o determinar la aplicabilidad universal de las máximas, o algo por el estilo.

Pero una vez que nos deshacemos de la ilusión formalista, de la reducción utilitarista - y esto significa resistir los engaños del modelo subyacente de validación racional- podemos ver que las fronteras de lo moral son una cuestión abierta; en efecto, incluso la aplicación correcta de un simple término puede ser un problema.

Podríamos decir fácilmente -un punto de vista que yo defenderíaque la atribución universal de personalidad moral es válida, y que trae consigo obligaciones que no podemos ignorar; pero que haya también otros ideales y metas morales -por ejemplo, la solidaridad universal, o la excelencia personal-que no podemos fácilmente coordinar con el universalismo y que incluso pueden entrar en conflicto con él. Decidir a priori cuáles son las fronteras de lo moral es, precisamente, ofuscar la cuestión de hacia dónde y en qué grado esto es así e, incluso, de hacerla imposible de responder coherentemente. ${ }^{11}$

Si las fronteras de lo moral no son difusas, sí podemos decir con Taylor que al menos no están todavía claras y completamente definidas,

${ }^{11}$ Ibid., p. 233. que son una cuestión a discutir, por lo que no podemos decidir a priori qué es lo válido y lo que no. En este sentido, más allá de mostrar que una creencia religiosa puede ser razonable -porque, además, tampoco es momento de reelaborar la teoría tayloriana del lenguaje de contrastes y la teoría de la racionalidad bajo las cuáles se evaluaría una creencia sin caer en el etnocentrismo-, baste con mostrar que los límites morales que imponen ciertos liberalismos deben ser ensanchados para que ese mismo liberalismo no resulte discriminatorio o etnocentrista. En esto está sustentado, a grandes rasgos, el multiculturalismo sui generis de Taylor: "Y así como todos deben tener derechos civiles iguales e igual derecho al voto, cualesquiera que sean su raza y su cultura, así también todos deben disfrutar de la suposición de que su cultura tradicional tiene un valor". ${ }^{12}$ No se trata únicamente de considerar a todos los agentes humanos igualmente dignos, sino de considerar que todos tienen, al menos prima facie, algo interesante y razonable que aportar. Las religiones, en consecuencia, con toda su carga de historia y de tradiciones, también. Esto no quiere decir que, de antemano, afirmemos cualquier identidad como válida o razonable, o que creamos en

${ }^{12}$ Charles Taylor, en Gutman, El multiculturalismo..., op. cit., p. 110. 
los dioses de los demás, o que pensemos que las religiones son verdaderas, sino únicamente que debemos considerar esta "apertura a la trascendencia", como decíamos hace unos momentos, como algo potencialmente razonable y racional. Y no solamente eso, sino que además de considerar el discurso del creyente como algo potencialmente razonable, hay también que considerar su identidad como una identidad potencialmente válida en el contexto de una sociedad moderna. ¿Qué quiere decir esto en el ámbito político?

\section{El reconocimiento y el comunitarismo}

Aparece ahora una nueva dimensión que, si bien estaba desde el comienzo en el trasfondo de nuestro discurso, ahora aparece como tema. ¿Cómo se inserta la identidad religiosa en la esfera pública? ¿Cómo integrarla en la sociedad secular? ¿Puede jugar algún papel en la política?

Podríamos comenzar con el siguiente argumento de Taylor:

Una teoría consecuencialista, incluso una que haya ido más allá del utilitarismo, sería todavía una cama de Procusto. Haría, una vez más, imposible para nosotros comprender del todo las diversas facetas de nuestro pensamiento moral y polí- tico. Y podría inducirnos a pensar que tendríamos el derecho de ignorar algunas demandas porque no caen dentro de nuestro modo favorecido de cálculo. Una meta-ética de este tipo obstaculiza el pensamiento. Nuestro pensamiento político necesita liberarse tanto de la mano muerta de la tradición epistemológica, como del monismo utópico del pensamiento radical, para así poder dar cuenta de la real diversidad de los bienes que reconocemos. ${ }^{13}$

En este sentido, Taylor propone un multiculturalismo no utilitarista y comprensivo, que reconozca toda identidad. Para ello, es necesario dejar en claro que todas las identidades deben ser por principio reconocidas en razón de que, dejando fuera una tradición epistemológica que achate los horizontes de la racionalidad, podemos ver que las expresiones y las identidades religiosas pueden aportar contenidos y bienes en la formación de la identidad comunitaria. ${ }^{14}$

${ }^{13}$ Charles Taylor, "The Diversity of Goods", op. cit., p. 247.

${ }^{14}$ Soy consciente de la dificultad de afirmar que las religiones pueden aportar contenidos morales valiosos a la sociedad; sobre todo en el ámbito público y en relación con la tradición epistemológica de las ciencias. Por lo pronto, remito a Fischer, Ernst Peter, “'Science Doesn't Tremble': The Secular Natural Sciences and the Modern Feeling for Life", en Joas, Hans y Klaus Wiegandt (eds.), Secularization and the World Religions, 2009, Liverpool, Liverpool University Press, trad. Alex Skinner. 
NOTAS

El objetivo de garantizar la libertad religiosa no sería, desde la filosofía de Taylor, únicamente lograr una convivencia pacífica entre las diversas expresiones identatarias, sino tener una sociedad que colabore con el crecimiento y la promoción de esas identidades.

Nuestra identidad se moldea en parte por el reconocimiento o por la falta de éste; a menudo, también, por el falso reconocimiento de otros, y así, un individuo o un grupo de personas puede sufrir un verdadero daño, una auténtica deformación de la gente o la sociedad que lo rodean le muestran, como reflejo, un cuadro limitativo, o degradante o despreciable de sí mismo. El falso reconocimiento o la falta de reconocimiento pueden causar daño, pueden ser una forma de opresión que subyugue a alguien en un modo de ser falso, deformado y reducido". ${ }^{15}$

Precisamente éste es un punto importantísimo para comprender el pensamiento de Taylor: su preocupación por el reconocimiento no es casual. No es por una necesidad procedimental o formal, no es una simple cuestión de derechos y garantías individuales, sino que la necesidad de reconocimiento remite a una noción antropológica de ser humano.

${ }^{15}$ Charles Taylor, en Gutman, op. cit., p. 53-4.
En su ensayo "Atomism” (“El atomismo"), Taylor emprende una seria crítica en contra de la tradición liberal que parte de Hobbes y de Locke. Para Taylor, tanto Hobbes como Locke comparten la doctrina de la primacía de los derechos:

Las teorías de la primacía de los derechos aceptan el principio de atribución a los hombres de derechos que valen incondicionalmente, es decir, valederos para los hombres como tales. Pero rechazan esa incondicionalidad para un principio de pertenencia u obligación. Antes bien, nuestra obligación de pertenecer a una sociedad o sostenerla, y obedecer a sus autoridades, se considera derivada; se nos impone de manera condicional, en virtud de nuestro consentimiento o por el hecho de ser ventajosa para nosotros". ${ }^{16}$

Las doctrinas atomistas, que son en general teorías del contrato social, han sido el fundamento del individualismo moderno, que concibe la obligación del individuo hacia la sociedad civil como algo que solamente vincula en la medida en que convenga a tal individuo. Por otra parte, obligación de la sociedad es garantizar los derechos inalienables de los individuos. El atomismo, en cierto

${ }^{16}$ Charles Taylor, “Atomism”, en Philosophical Papers 2. Philosophy and the Human Sciences, 1985, Cambridge, Cambridge University Press, p. 188. 
sentido, afirma la autosuficiencia del hombre aislado, del individuo. En este punto, Taylor se separa de un tipo de tradición liberal en la que el individuo y sus derechos son el fundamento de la sociedad y se pronuncia, más bien, por una suerte de comunitarismo. El argumento de Taylor es, más o menos, el siguiente: afirmar la primacía del individuo y de sus derechos es sostenible sobre una base antropológica, sobre una cierta noción de ser humano. Pensar que el individuo y el hombre aislado es autosuficiente en el sentido de vital y biológico no es algo que estuviera en la cabeza de Hobbes y de Locke, a pesar de que estos teóricos sostuvieran, muchos de ellos, un hipotético estado primitivo de naturaleza en la que el ser humano vivía aislado y luego hizo un contrato social. Sostener la primacía de los derechos, entonces, dice Taylor, quiere decir otra cosa. Quiere decir que hay una capacidad humana que debe respetarse por considerarse valiosa: querer garantizar los derechos como primer paso para una sociedad implica mucho más que la simple posibilidad de ejercerlos. "La intuición de que los hombres tienen el derecho a la vida, a la libertad, a la profesión irrestricta de sus convicciones, al ejercicio de sus creencias morales o religiosas, no es sino otra faceta de la idea de que la forma de vida caracterizada por estas capa- cidades específicamente humanas nos impone respeto. Los seres con esas capacidades exigen nuestro respeto porque éstas son de especial significación para nosotros; tienen una jerarquía moral especial". ${ }^{17}$ Así-y aquí continúo con el razonamiento de Taylor-, habría una inconsistencia -un hecho irracional- en pretender atribuir derechos sin afirmar también que el ejercicio de los mismos tiene un valor especial dentro de una jerarquía moral.

Esto no significa que valoremos solamente el derecho a ejercer ciertas capacidades o a tener ciertas creencias, sino que "también dice que debo llegar a ser el tipo de agente capaz de una auténtica convicción, que debo ser fiel a mis convicciones y no vivir una mentira o un autoengaño por temor o con vistas a obtener una ventaja, que en ciertas circunstancias debo ayudar a fomentar esa capacidad en otros, que debo educar a mis hijos con ese objeto, que no debo inhibirla en otros induciéndoles a una complacencia fácil y superficial, etc." ${ }^{18}$ Por ello, afirmar un derecho es, también, afirmar el valor de ejercerlo y el valor de llevar una vida de acuerdo con ese ejercicio. Justamente aquí, Taylor hace la crítica al atomismo, pues el individuo libre o agente moral no puede ejercer esos derechos y no

${ }^{17}$ Ibid., p. 193.

${ }^{18}$ Ibid., p. 194. 
NOTAS

pude realizar sus capacidades humanas en sentido pleno si no es perteneciendo a una comunidad y a un tipo determinado de cultura.

Sostengo -señala Taylor- que el individuo libre y occidental es lo que es sólo en virtud de la totalidad de la sociedad y la civilización que lo produjeron y lo nutren; que nuestras familias sólo pueden educarnos en la capacidad y las aspiraciones aludidas porque pertenecen a esa civilización, y que una familia aislada y fuera de ese contexto -la verdadera familia patriarcal de antañoesa una especie muy diferente que nunca tendía hacia esos horizontes. Y sostengo, por último, que todo esto genera una significativa obligación de pertenencia en quien quiera afirmar el valor de esa libertad, e incluyo en este grupo a todos los que deseen afirmar derechos a dicha libertad o en su beneficio. ${ }^{19}$

La comunidad es, pues, anterior a los derechos.

La identidad, entonces, no se logra en el aislamiento, sino que es siempre dialógica. Es necesario lograr conformar una sociedad que afirme y reconozca la identidad personal para que ésta pueda desarrollarse a través del tiempo. No nos constituimos a nosotros mismos de manera completamente autónoma: "siempre definimos

${ }^{19}$ Ibid., p. 206. nuestra identidad en diálogo con las cosas que nuestros otros significantes desean ver en nosotros, y a veces en lucha con ellas". ${ }^{20}$ De este modo, la necesidad de reconocimiento de la identidad está fundamentada, para Taylor, en su comunitarismo, que consiste en afirmar que el ser humano no puede desarrollar sus capacidades humanas de manera aislada, sino que para ello necesita de los otros. "La comunidad -dice Pérez Barahona-, ofrece los marcos valorativos adecuados donde se desarrolla el lenguaje que posibilita dotar de sentido las distintas valoraciones morales". La alteridad social es, pues, necesaria para que tenga sentido la afirmación y la garantía de los derechos, lo que se consigue únicamente cuando la comunidad misma propicia el desarrollo de las diversas identidades que la constituyen.

\section{La esfera pública y las identidades religiosas}

El reconocimiento de las identidades culturales, entre ellas las religiosas, será mucho más que garantizar el derecho a su ejercicio, sino permitir que se expresen bajo sus propios términos, por supuesto, siempre garantizando que se preserve el principio de no dañar a terceros. Taylor entiende

\footnotetext{
${ }^{20}$ Taylor, en Gutman, op. cit., p. 63.
} 
por "esfera pública" "un espacio común donde los miembros de la sociedad se encuentran, a través de una cierta variedad de medios de comunicación (impresos, electrónicos) y también en reuniones cara a cara, para discutir asuntos de interés común y, de este modo, ser capaces de de formar una opinión común sobre ellos". ${ }^{21}$ Por eso, las instituciones políticas y sociales en las que se fundamenta lo social y el espacio público son claves para el desarrollo y afirmación de nuestra propia identidad: si allí formaremos una opinión común sobre los asuntos de todos, entonces el Estado liberal debe garantizar que los grupos religiosos se manifiesten libremente, sin obligarlos a fracturar su identidad.

Esto ha sido visto ya incluso por pensadores como Jürgen Habermas, quienes han reconocido abiertamente que la modernidad no tendría por qué terminar en una secularización, o que el mismo significado de secularización no necesariamente significa la reducción de lo religioso a lo privado o incluso su eliminación, sino que la modernidad significa, también, una era post-secular en la que las religiones no se conciben ya como enemigas de la Ilustración o de la modernidad. "El Estado liberal garantiza una libertad igual en la prác-

\footnotetext{
${ }^{21}$ Charles Taylor, "La política liberal y la esfera pública”, en Argumentos filosóficos, 1997, Barcelona, Paidós, trad. Fina Birulés Bertrán, p. 337.
}

tica religiosa no sólo para mantener la paz y el orden, sino también por el motivo normativo de proteger la libertad de culto y la libertad de conciencia de cada cual. Por eso no debe exigir a sus ciudadanos religiosos algo que sea incompatible con una existencia vivida auténticamente 'desde la fe", ${ }^{22}$ e incluso: "El Estado liberal también debe esperar de sus ciudadanos seculares que, en su condición de ciudadanos del Estado, no consideren las manifestaciones religiosas como algo meramente irracional". ${ }^{23}$ Esta afirmación de la libertad y del ejercicio de las creencias religiosas tiene que ver con el funcionamiento de una sociedad como el medio en el cual se desenvuelven y se realizan los individuos, en la medida en que los individuos creyentes necesitan de un contexto social que les permita ejercer su actividad como agentes racionales y creyentes. Como señala Fernando Pliego, "cuando los individuos no articulan sus creencias bajo el principio de libertad de creencia plena ni tampoco tienen el derecho de presentarlas en la esfera pública de la sociedad sin peligro de coacción alguna, entonces tales individuos no pueden participar activamente en la construcción argumentativa de las

${ }^{22}$ Jürgen Habermas, "La conciencia de lo que falta", en Carta al Papa. Consideraciones sobre la fe, 2009, Barcelona, Paidós, trad. Bernardo Moreno Carrillo, p. 72.

${ }^{23}$ Ibid., p. 73-4. 
NOTAS

instituciones propias de una sociedad democrática". ${ }^{24}$ Afirmar la identidad religiosa en la esfera pública es clave para que este sector de la sociedad pueda participar activamente en la construcción dialógica, no solamente de sí mismos, sino de las diversas identidades con las que convive.

En este sentido, las posiciones de Habermas se acercan significativamente al multiculturalismo de Charles Taylor quien, más allá de creer en una inconmensurabilidad de las culturas o en el relativismo cultural, afirma que el momento de comprensión de la alteridad y de su afirmación prima facie como una posibilidad valiosa de realización humana, inclui-

${ }^{24}$ Fernando Pliego, "Convicciones religiosas y bien público", en Conspiratio, 2010, núm. 8, año II, p. 71. das por supuesto las identidades religiosas, son imprescindibles para el desarrollo de una humanidad coherente consigo misma e, incluso, para todo liberalismo que quiera ser consecuente con sus propias premisas. Una sociedad secular no es, pues, una sociedad en la que las creencias religiosas han decaído o se llevan al ámbito privado, sino una sociedad en la cual las creencias religiosas pueden aparecer libremente y sin trabas en el espacio público, en convivencia con todas las otras manifestaciones culturales e identatarias para generar, en común, las perspectivas racionales que deberían guiar a la sociedad. ${ }^{25}$

\footnotetext{
${ }^{25}$ Esto se debe, en buena medida, a lo que el mismo Taylor señala, creer en Dios se ha vuelto una opción entre muchas otras: "el cambio que intento definir y trazar es el que nos llevó de una sociedad en la cual era virtualmente imposible creer en Dios, a una en la cual la fe, aún para el más firme creyente, es una posibilidad humana entre otras", Charles Taylor, A Secular Age, 2007, Cambridge Mass., The Belknap Press of Harvard University Press, p. 3.
} 